\title{
The Design of Intelligent Logistics System Based on Internet of Things \\ Jianguo $\mathrm{Xu}^{1, \mathrm{a}^{*}}$ \\ ${ }^{1}$ Yangtze University College of Technology \& Engineering, Jingzhou 434020, China \\ a93323822@qq.com
}

\begin{abstract}
Keywords: Intelligent logistic system; Internet of thing; RFID; ZigBee; A10
\end{abstract}
\begin{abstract}
With the rapid development of Internet technology, as well as interoperability of RFID technology, the popularity of ZigBee technology, things and objects can be achieved, this article discusses the design of smart logistic system of things Based on A10 Andriod-based system platform. System hardware consists of A10 platform, RFID modules, ZigBee modules, servers, mobile terminal, etc. The ZigBee sensors within the warehouse can monitor multiple points environment (including temperature, humidity, smoke alarm, PIR), RFID module can manage the track of goods storage. ZigBee coordinator and RFID reader module collected data and transferred to the A10 platform real-time processing.

Then the data uploaded to the web server through the internet, users can obtain relevant information through the mobile terminal or Web. Experimental results showed this approach could reduce dependence on staff greatly and have good effect in actual application.
\end{abstract}

\section{Introduction}

At present, many logistics service enterprises are still in the traditional, simple service, consider the logistics management as transportation, warehousing, distribution and processing separate part, independently managed. Many logistics enterprise informatization level is low, could not provide information sharing for customers, so that companies have been slow to respond, inefficient [1].

The development of the Internet of things technology is driving the change of Chinese wisdom logistics. RFID technology, ZigBee technology gained popularity, so that the interconnection of things can be achieved, will give enterprise logistics systems, such as environment monitoring systems provide a platform [2,3]. On this basis, this paper introduces the design of intelligent logistics system based on Internet of things. The system can real-time monitoring goods information and environmental security, reduce the dependence on staff.

\section{The Hardware Design of the System}

System Overall Framework. The Intelligent logistic system based on Internet of thing architecture diagram shown in Fig. 1, the system consists of the A10 platform(Andriod-based), RFID modules, ZigBee modules, servers, mobile devices, etc. The ZigBee sensors within the warehouse can monitor multiple points environment (including temperature, humidity, smoke alarm, PIR), RFID module can manage the track of goods storage. ZigBee coordinator and RFID reader module collected data and transferred to the A10 platform real-time processing via USB. Then the data uploaded to the web server through the internet, users can obtain relevant information through the mobile terminal or Web. 


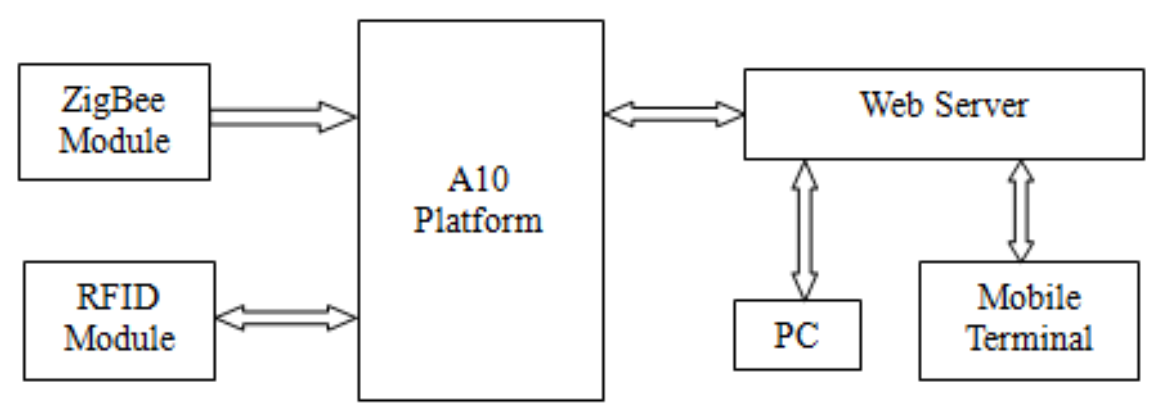

Figure 1. The system hardware block diagram

The A10 Platform Based On Andriod. The system hardware platform uses A10 processor core, the software uses Andriod system. The platform is very rich of peripheral expansion circuit, the output displayed can use LCD display, LVDS interfaces, HDMI, VGA and so on. The platform as a transit hub of data flow, is a collection point of data, mainly collect environmental information of warehouse (including temperature, humidity, smoke alarm, PIR)、 real-time dynamic data of warehouse entrance, splice the data into JSON and then upload to the server via RJ45 interface[4,6].

ZigBee Module. The ZigBee module includes a coordinator and sensors, the sensors are placed in different positions of the logistics warehouse, collect multi-point environmental information of warehouse(including temperature, humidity, smoke alarm, PIR). After the success of the networking, the coordinator uses polling to receive sensor data, and transfers the data to the hardware platform.

The RFID Module. The module can manage the goods receipt of logistics terminal, and the goods in storage warehouse when transit. When the delivery clerk at the terminal recipient, they can write the relevant information to the electronic tag through software and paste the tag on the surface of the goods. The RFID module is consist of RFID reader module and the electronic tag module[8]. Electronic tag attached to the surface of the goods and stored the information of the sender, recipient, order number, cell phone number and so on. Reader module is installed in the entrance to the warehouse, can receive the data of the electronic tag when the goods in and out of storage and access to the electronic tag identification zone, transfers the real-time data to the hardware platforms and server.

Server. The server is mainly to complete the following aspects:

1) Receiving environment monitoring data from the system platform and the information of goods in to the storage.

2) Sending the information to the Web client or mobile terminal via the Internet.

The Mobile Terminal. After the mobile terminal runs the client APP program, will continuously sends the Web server data request, the server returns the corresponding data. The APP program will shows APP page after receive the data. The users can browse, query, and ultimately warehouse real-time monitoring of the environment and the cargo storage information in real-time queries.

\section{The Software Design of the System}

The Server Configuration and Implementation. Using MVC architecture to write code for WEB. JSP dynamic display the user's query results after the order number in the View layer, including sender, recipient, goods and warehouse status. The warehouse manager can view the information of the warehouse after login the management, including temperature, humidity, storage and inventory list, in which the temperature and humidity using graph to display. The system administrator can 
view all the information hub of a province or a transit point by querying, and can modify the information of a transit point. Control operation of the database layer is required for each table to write SQL statements and interfaces for the Model layer calls. Model layer is to provide a method for each logical processing and data Servlet files require.

The data transfer between the server and the client Andrews uses JSON. The Android client requests the server to accept the request and then stores the data into the database. Particularly, the warehouse management module draws a JSP graph of temperature and humidity from the sensor and then shows in the module of warehouse administrator.

The Design of A10 Platform. A10 server is a bridge connecting the bottom and web servers. It collects the data from ZigBee coordinator, and then decodes the received data, after processing. Requesting server, and store the data in the web database.

The work of A10 server mainly divided into three parts: read and write of JNI serial; Activity set the serial port and baud rate of ZigBee coordinator; and set the information of current transfer station. The service always runs in the background for data reception, decoding, and transmission package.

The Application Design for the Mobile Terminal. The application is a software app running on more than android2.3 for the mobile terminal. The software App mainly to complete two functions: environmental monitoring, and warehouse data browsing [7,9]. It uses the classic MVC architecture of android, can effective separate the interface and back-end logic. Use Http protocol, JSON objects, Java tunnel communications, interact with the network database. Asynchronous request, then present the data to the user.

\section{System Test}

Database Connection Test. The Fig. 2 is testing receive data from the A10 platform, the data has been successfully resolved to JSON. Here have two data, one data type is infrared and the value is 0.8 , time of $3: 45: 15$, another data type is smoke and the value is 0.3 , time is $3: 45: 15$. Then data is sent to the database and storage.

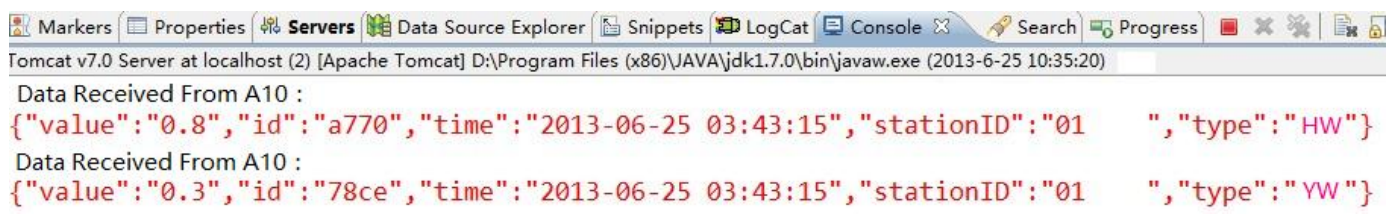

Figure 2. The test of receive data

After a short time, the database has more than 1,800 pieces of data (temperature, humidity, infrared, smoke), shows that the real-time record condition is good.

External Request Test. When the server receive a request from the mobile terminal to send data, at the control to postback the data correspond with. The data is sent in the form of JSON, including transit place, time, lights status information. This data is sent back to the mobile phone APP program is used to present to the user.

The Mobile Terminal Test. After a successful login, users could get the information of goods according to the order number. Users can enter their order number on the left, the right side displays the status information of goods. In the status information page, users can view all the transit route of of the order, as well as the current status information. 
In addition to the completion the test of the storage, the sensor detect the smoke density and temperature and humidity changes can occur obvious transition in a short time, indicating that the system environmental monitoring functions operate normally.

\section{Summary}

Based on the research of RFID technology and ZigBee technology, this paper designed a Intelligent Logistics System Based on Internet of Things, the system mainly achieve the following functions:

1 After the success of the networking, the coordinator uses polling to receive sensor data, and transfers the data to the A10 hardware platform.

2 Design the application software, when the delivery clerk at the terminal recipient, they can write the relevant information into the computer, and write the serial number to the electronic tag, with the electronic tag can facilitate the identification of goods in transit.

3 On the basis of automatically read the electronic tag paste the tag on the surface of goods to achieve the automatic goods out of storage, reducing the dependence on staff.

\section{Acknowledgments}

This work was supported by Project of Hubei Province Education Science Planning 2015 (2015GB165).

\section{References}

[1] Zhang Z R, Wang D W, Zhang Z G, et al. Study on CRE Logistics Management Mode of Operation Based on The Internet of Things[J]. Journal of Dalian Jiaotong University, 2014.

[2] Luoh L. ZigBee-based intelligent indoor positioning system soft computing [J]. Soft Computing, 2014, 18(3):443-456.

[3] Saab S S, Nakad Z S. A Standalone RFID Indoor Positioning System Using Passive Tags [J]. IEEE Transactions on Industrial Electronics, 2011, 58(5):1961-1970.

[4] Butler M. Android: Changing the Mobile Landscape [J]. IEEE Pervasive Computing, 2011, 10(1):4-7.

[5] Jiang X, Gao J S. Fault diagnosis system for industrial machinery based on embedded HTML5[C]// IEEE International Conference on Information and Automation. 2013:653-658.

[6] Android G. GOOGLE ANDROID », Web Design e Internet [J]. Google Android.

[7] Ongtang M, Mclaughlin S, Enck W, et al. Semantically Rich Application-Centric Security in Android $[\mathrm{C}] / /$ Computer Security Applications Conference. IEEE Computer Society, 2009:340-349.

[8] Finkenzeller K. RFID Handbook: Fundamentals and Applications in Contactless Smart Cards and Identification, Second Edition [M]// RFID Handbook: Fundamentals and Applications in Contactless Smart Cards and Identification. John Wiley \& Sons, Inc. 2003.

[9] Heilmann C. Beginning JavaScript with DOM Scripting and Ajax [M]. Apress, 2006.

[10] Li L. Application of the Internet of Thing in Green Agricultural Products Supply Chain Management $[\mathrm{C}] / /$ International Conference on Intelligent Computation Technology and Automation. 2011:1022-1025. 